\title{
An implicit parallel UGKS solver for flows covering various regimes
}

\author{
Dingwu Jiang ${ }^{1}$, Meiliang Mao ${ }^{1 *}$, Jin $\mathrm{Li}^{1}$ and Xiaogang Deng ${ }^{2}$
}

\author{
* Correspondence: \\ mml219@163.com \\ ${ }^{1}$ Computational Aerodynamics \\ Institute, China Aerodynamics \\ Research and Development Center, \\ Mianyang 621000, China \\ Full list of author information is \\ available at the end of the article
}

\begin{abstract}
This paper presents an engineering-oriented UGKS solver package developed in China Aerodynamics Research and Development Center (CARDC). The solver is programmed in Fortran language and uses structured body-fitted mesh, aiming for predicting aerodynamic and aerothermodynamics characteristics in flows covering various regimes on complex three-dimensional configurations. The conservative discrete ordinate method and implicit implementation are incorporated. Meanwhile, a local mesh refinement technique in the velocity space is developed. The parallel strategies include MPI and OpenMP. Test cases include a wedge, a cylinder, a 2D blunt cone, a sphere, and a X38-like vehicle. Good agreements with experimental or DSMC results have been achieved.

Keywords: Unified gas kinetic scheme, Conservative discrete ordinate method, Implicit algorithm, Mesh refinement, MPI, OpenMP, Application
\end{abstract}

\section{Introduction}

During the reentry process, vehicles may encounter different flow regimes such as free molecular, transitional, near continuum, and continuum regime. The determination of aerodynamic forces and heat loads has great impact on the design of vehicles [1]. In the non-continuum regimes, traditional macroscopic methods, such as Euler, Navier-Stokes and Burnett equations, may become invalid. The following methods are mainly used for the non-equilibrium flow simulations. The first kind of method is based on probabilistic modeling. The most popular one is the direct simulation Monte Carlo (DSMC) method. DSMC was first proposed by Bird [2] more than half a century ago. It follows the evolution of representative particles with uncoupled transport and collision process. The DSMC has been fully validated for providing physical solutions through its comparison with the experiments measurements [3, 4]. It has played a key role in the design and flight analysis of vehicles in the rarefied environment. Some of the most cited DSMC codes in literature are DS2V/3 V [5], DAC [6], SMILE [7], MONACO [8], and DSMCFOAM [9]. The main differences among these codes are in the treatment of collision selection methods and mesh topology.

Another kind of approach is the deterministic method. Deterministic method mainly concerns the Boltzmann equation. Due to the complexity of the Boltzmann collision term, researchers usually choose the simplified collision model, such as BGK model [10], Shakhov model [11], Rykov model [12]. Titarev [13] has developed an implicit 
solver named Nesvetay-3D on unstructured mesh. Three-dimensional TVD method is applied for the numerical discretization. Both spatial and velocity mesh decomposition are used in the parallelization. A total number of $6.9 \times 10^{9}$ mesh points in the six-dimensional space is used for the supersonic flow simulation around a re-entry space vehicle. Wadsworth [14] has developed a parallel, finite volume 2D/axisymmetric code SMOKE which is based on conservative numerical schemes developed by Mieussens [15]. In Baranger's team, a 3D code [16] has been used in the past years for rarefied flow simulations. This code can handle polyatomic gases. It uses block structured mesh and hybrid parallelization, i.e., space domain decomposition with MPI and inner parallelization with OpenMP. Furthermore, the code is equipped with velocity mesh refinement technique which improves the code in both CPU time saving and memory storage. Li's team has developed a 3D code based on the model equation with the name gas-kinetic unified algorithm (GKUA) [17, 18]. Three-dimensional hypersonic flows around sphere and spacecraft with different Knudsen numbers and Mach numbers have been studied. The total six-dimensional mesh for a complex wing-body configuration reaches $7.3 \times 10^{11}$ and 23,800 CPU cores [19] have been used in the computation.

However, the above deterministic methods share a common feature. They decouple the particle transport and collision. Therefore, the cell size and time step in these numerical schemes are limited by the particle mean free path and mean collision time in order to provide accurate numerical solutions. When the flow regime is close to continuum or near continuum, the time step and cell size limitations are rather severe and make these methods extremely time-consuming and inefficient.

Another distinguishable deterministic method, which is named unified gas kinetic scheme (UGKS), was proposed by Xu et al. [20-22]. UGKS is a multi-scale method with coupled particle transport and collision in its numerical flux modeling. It is based on an integral solution of the gas-kinetic model equation. It can recover the flow physics from the kinetic particle transport and collision to the hydrodynamic wave propagation. Moreover, the time step is determined only by the CFL condition, which is not limited by the mean collision time. So the scheme becomes more efficient in various flow regimes, especially when the local Knudsen number is low. Applying UGKS to analyze aerodynamic and aerothermodynamics on flying vehicles in near space flight is our long term objective.

This paper is organized in the following. Section 2 is about the introduction of UGKS and some techniques to accelerate convergence. Section 3 is a simple description of the framework. Section 4 is some $2 \mathrm{D}$ and $3 \mathrm{D}$ validation test cases. The last section is the conclusion.

\section{Method}

\subsection{Unified gas kinetic scheme}

The three-dimensional Shakhov model equation [11],which can give the correct Prandtl number, in non-dimensional form reads

$$
f_{t}+u f_{x}+v f_{y}+w f_{z}=\frac{f^{+}-f}{\tau}
$$

where the free-stream parameters density $\bar{\rho}_{\infty}$, velocity $\bar{U}_{\infty}$, viscosity coefficient $\bar{\mu}_{\infty}$ and 
the characteristic length $\bar{L}$ are used and the resulting non-dimensional variables are given by.

$$
\begin{aligned}
& (x, y, z)=(\bar{x}, \bar{y}, \bar{z}) / \bar{L}, t=\bar{t} /\left(\bar{L} / \bar{U}_{\infty}\right),(u, v, w)=(\bar{u}, \bar{v}, \bar{w}) / \bar{U}_{\infty}, \rho=\bar{\rho} / \bar{\rho}_{\infty} \\
& p=\bar{p} /\left(\bar{\rho}_{\infty} \bar{U}_{\infty}^{2}\right), \tau=\bar{\tau} /\left(\bar{L} / \bar{U}_{\infty}\right), \mu=\bar{\mu} / \bar{\mu}_{\infty}, \lambda=\bar{\lambda} /\left(1 / \bar{U}_{\infty}^{2}\right), f=\bar{f} /\left(\bar{\rho}_{\infty} / \bar{U}_{\infty}^{3}\right) \\
& f^{+}=\bar{f}^{+} /\left(\bar{\rho}_{\infty} / \bar{U}_{\infty}^{3}\right), g_{M}=\bar{g}_{M} /\left(\bar{\rho}_{\infty} / \bar{U}_{\infty}^{3}\right) .
\end{aligned}
$$

$f^{+}$can be given in the form, $f^{+}=g_{M}+g^{+}$

Here $g_{M}$ is the Maxwellian distribution function

$$
g^{+}=g_{M}(1-\operatorname{Pr}) \vec{c} \cdot \vec{q}\left(c^{2} / R T-5\right) /(5 p R T)
$$

and $\vec{c}=\vec{u}-\vec{U}$ is the peculiar velocity. $T, \vec{q}$, Pr are the temperature, heat flux and Prandtl number, respectively.

The relations between conservative variables $\rho, \rho U, \rho V, \rho W, \rho E$ with the probability density function is

$$
(\rho, \rho U, \rho V, \rho W, \rho E)^{\mathrm{T}}=\int \boldsymbol{\psi}^{\mathrm{T}} f d \Xi
$$

where $\boldsymbol{\psi}^{\mathrm{T}}=\left(1, u, v, w, 1 / 2\left(u^{2}+v^{2}+w^{2}\right)\right)^{\mathrm{T}}$ is vector of moments and $d \Xi=d u d v d w$ is the volume element in the phase space.

Integrating Eq. (1) in the volume element we can get

$$
\begin{aligned}
& \frac{\partial \mathbf{Q}}{\partial t}+\frac{\partial \mathbf{F}}{\partial x}+\frac{\partial \mathbf{G}}{\partial y}+\frac{\partial \mathbf{H}}{\partial y}=0 \\
& \mathbf{F}=\int u f \boldsymbol{\Psi}_{\alpha} d \Xi \quad \mathbf{G}=\int v f \boldsymbol{\Psi}_{\alpha} d \Xi \mathbf{H}=\int w f \boldsymbol{\Psi}_{\alpha} d \Xi
\end{aligned}
$$

where the conservation constraint or compatibility condition in the following form has been used

$$
\int\left(f-f^{+}\right) \boldsymbol{\Psi}_{\alpha} d \Xi=0, \alpha=1,2,3,4,5
$$

For curvilinear coordinate system, applying the finite volume method eq. (3) goes to

$$
\begin{aligned}
& \Delta \mathbf{Q}=-V^{-1} \int_{t^{n}}^{t^{n+1}}\left[\begin{array}{l}
(\mathbf{J} \cdot \mathbf{S})_{i+1 / 2, j, k}-(\mathbf{J} \cdot \mathbf{S})_{i-1 / 2, j, k} \\
+(\mathbf{J} \cdot \mathbf{S})_{i, j+1 / 2, k}-(\mathbf{J} \cdot \mathbf{S})_{i, j-1 / 2, k} \\
+(\mathbf{J} \cdot \mathbf{S})_{i, j, k+1 / 2}-(\mathbf{J} \cdot \mathbf{S})_{i, j, k-1 / 2}
\end{array}\right] d t \\
& \mathbf{J}=\mathbf{F i}+\mathbf{G j}+\mathbf{H k}
\end{aligned}
$$

where $V$ is the cell volume, $\mathbf{S}$ and $\mathrm{J}$ are the cell face vectors and flux vectors, respectively.

The flux across a cell interface is based on the integral solution of the model equation. Discontinuous spatial reconstruction with nonlinear limiter is used to introduce artificial dissipation for UGKS once the scheme becomes a shock capturing method when the dissipative flow structure cannot be well resolved by the cell size. Details can be found in [20]. In this paper, we use van Leer limiter in the reconstruction. Due to the discreteness of the velocity space, numerical quadrature should be used to calculate various integrals. In this paper, composite Newton-Cote's $(N-C)$ quadrature is adopted.

The Rykov model [12] for diatomic gases is also implemented in our UGKS code package. The corresponding details are omitted. 


\subsection{Conservative discrete ordinate method [23]}

The compatibility condition Eq. (4) is the basis for the governing Eq. (3). But once the DOM is introduced and the velocity space is discretised, Eq. (4) no longer holds and becomes

$$
\int\left(f-f^{+}\right) \Psi_{\alpha} d \Xi=\operatorname{Err}(N-C)
$$

Here Err is the numerical error introduced by the numerical quadrature. Err can be reduced by increasing the velocity space mesh in a certain extent but will finally stay in some level, which is determined by the intrinsic nature of numerical quadrature.

This numerical error results in a source term in the governing Eq. (5). The source term can be expressed in the form $\int_{t^{\zeta}}^{t^{\zeta+1}}\left[\frac{1}{\tau} \operatorname{Err}(N-C)\right] d t$

Define

$$
\mathbf{S S}=\frac{1}{\Delta t} \int_{t^{\zeta}}^{t^{\zeta+1}}\left[\frac{1}{\tau} \operatorname{Err}(N-C)\right] d t \approx\left[\frac{1}{\tau} \operatorname{Err}(N-C)\right]^{\zeta+1}
$$

Here $\Delta t$ is the marching time step. The five components of $\mathbf{S S}$ correspond to the governing equations of mass, momentum in the $\mathrm{x}, \mathrm{y}$ and $\mathrm{z}$ directions and the energy, respectively. After some simple derivations we can get

$$
\tau=\frac{\mu}{p \operatorname{Re}_{\infty}} \propto \frac{K n_{\infty}}{M_{\infty}} \frac{\mu}{p}
$$

From Eq. (7) and Eq. (8) we can see that SS is related to free-stream condition and numerical quadrature.

In order to eliminate the numerical source term completely, we introduce CDOM proposed by Titarev [24] into UGKS,

$$
\iiint \frac{f^{+}-f}{\tau} \psi_{1}^{\mathrm{T}} d u d v d w=\frac{1}{\tau}\left(0,0,0,0,0,-2 / 3 q_{x},-2 / 3 q_{y},-2 / 3 q_{z}\right)^{\mathrm{T}}
$$

where

$$
\begin{aligned}
& \psi_{1}^{\mathrm{T}}=\left(1, u, v, w, \frac{1}{2}\left(u^{2}+v^{2}+w^{2}\right), \frac{1}{2}(u-U) \vec{c}^{2}, \frac{1}{2}(v-V) \vec{c}^{2}, \frac{1}{2}(w-W) \vec{c}^{2}\right)^{\mathrm{T}} \\
& \vec{c}^{2}=(u-U)^{2}+(v-V)^{2}+(w-W)^{2}
\end{aligned}
$$

The first five equations in (9) represent conservation of mass, momentum and energy during collision process. In discretised velocity space, the multiple integral is replaced by numerical quadratures. If the equilibrium distribution function remains in the form given in section 2.1, Eq. (9) no longer holds due to numerical error of quadratures. In other words, the conservation property will not be maintained.

Substituting the expression $\iiint f \psi_{1}^{\mathrm{T}} d u d v d w=\left(\rho, \rho U, \rho V, \rho W, \rho E, q_{x}, q_{y}, q_{z}\right)^{\mathrm{T}}$ into Eq. (9) we can get a new Eq. (10), which can be solved by the Newton iteration method. An initial guess equals to $\left(\rho, U, V, W, \lambda, q_{x}, q_{y}, q_{z}\right)$ is provided. Then a new group of variables, $\left(\rho^{\prime}, U^{\prime}\right.$, $\left.V^{\prime}, W^{\prime}, \lambda^{\prime}, q_{x}^{\prime}, q_{y}^{\prime}, q_{z}^{\prime}\right)$ can be got. 


$$
\sum f^{+} \psi_{2}^{\mathrm{T}}-\left(\rho, \rho U, \rho V, \rho W, \rho E, q_{x}, q_{y}, q_{z}\right)^{\mathrm{T}}=\left(0,0,0,0,0,-2 / 3 q_{x},-2 / 3 q_{y},-2 / 3 q_{z}\right)^{\mathrm{T}}
$$

where

$$
\begin{aligned}
\psi_{2}^{\mathrm{T}} & =\left(1, u, v, w, \frac{1}{2}\left(u^{2}+v^{2}+w^{2}\right), \frac{1}{2}\left(u-U^{\prime}\right) \vec{c}^{\prime 2}, \frac{1}{2}\left(v-V^{\prime}\right) \vec{c}^{\prime 2}, \frac{1}{2}\left(w-W^{\prime}\right) \vec{c}^{\prime 2}\right)^{\mathrm{T}} \\
\vec{c}^{\prime 2} & =\left(u-U^{\prime}\right)^{2}+\left(v-V^{\prime}\right)^{2}+\left(w-W^{\prime}\right)^{2}
\end{aligned}
$$

Here the symbol $\sum$ indicates that numerical quadratures are used. With the discrete $f^{+}$determined by the above group of variables, the conservation property holds and the numerical source term Err goes to machine zero, which has been validated in numerical experiments.

The UGKS in Section 2.1 has a second-order of accuracy. What we do in this section only changes the form of the heat flux modified equilibrium state. The spatial reconstruction and the evaluation of the numerical flux remain unchanged. Thus, CDOM does not affect the spatial accuracy and the coupling of particle transport and collision.

\subsection{Implicit UGKS [25]}

The governing equation in a physical control volume $(\mathrm{i}, \mathrm{j}, \mathrm{k})$, at velocity mesh point $\boldsymbol{u}_{l, m, n}=\left(u_{l}, v_{m}, w_{n}\right)$, is given by

$$
\frac{\partial f_{i, j, k, l, m, n}}{\partial t}+u_{l} \frac{\partial f_{i, j, k, l, m, n}}{\partial x}+v_{m} \frac{\partial f_{i, j, k, l, m, n}}{\partial y}+w_{n} \frac{\partial f_{i, j, k, l, m, n}}{\partial z}=\frac{\left(f_{i, j, k, l, m, n}^{+}-f_{i, j, k, l, m, n}\right)}{\tau}
$$

Define $\Delta f=f^{\zeta+1}-f^{\zeta}$ and $\Delta t=t^{\zeta+1}-t^{\zeta}$, then the implicit method reads

$$
\begin{aligned}
(1+\Delta t & \left.\frac{1}{\tau^{\zeta}}+\Delta t \cdot \mathrm{u}_{l, m, n} \nabla\right)(\Delta f)_{i, j, k, l, m, n}=\Delta t \cdot R_{i, j, k, l, m, n}^{\zeta} \\
R_{i, j, k, l, m, n}^{\zeta} & =-u_{l} \frac{\partial f_{i, j, k, l, m, n}^{\zeta}}{\partial x}-v_{m} \frac{\partial f_{i, j, k, l, m, n}^{\zeta}}{\partial y}-w_{n} \frac{\partial f_{i, j, k, l, m, n}^{\zeta}}{\partial z}+\frac{1}{\tau^{\zeta}}\left(f^{+}-f\right) \\
& =-R^{\prime}+\frac{1}{\tau^{\zeta}}\left(f^{+}-f\right)
\end{aligned}
$$

where $R^{\prime}$ is the evolving time averaged flux which can be written as

$$
R^{\prime}=\frac{\int_{0}^{\Delta t t} \sum_{i i=1}^{6} u_{\mathrm{n}} f_{p}(t) d t}{\Delta t t}
$$

where $u_{\mathrm{n}}=\boldsymbol{u}_{l, m, n} \cdot \boldsymbol{n}_{i i}$ and $\boldsymbol{n}_{i i}$ is the unit vector normal to the cell interface. The evolving time step $\Delta t t$ is different from the marching time step $\Delta t$. Based on some numerical experimental results, we propose in this paper the following principle to determine $\Delta t t$

$$
\Delta t t<\Delta t_{\min } / C F L
$$

where $\Delta t_{\text {min }}$ is the minimum time step in the whole field determined by the CFL condition. Eq. (12) can be rewritten in the following form

$$
\begin{aligned}
& \left(1+\Delta t \cdot \frac{1}{\tau^{\zeta}}\right)(\Delta f)_{i, j, k, l, m, n}+\frac{\Delta t}{\left|V_{i, j, k}\right|} \sum_{i i=1}^{6}\left(\mathbf{u}_{l, m, n} \cdot \mathbf{n}_{i i}\right) \cdot\left|S_{i, j, k, i i}\right| \cdot F F\left((\Delta f)_{i, j, k, l, m, n},(\Delta f)_{i 1, j 1, k 1, l, m, n}\right) \\
& =\Delta t \cdot R_{i, j, k, l, m, n}^{\zeta}
\end{aligned}
$$




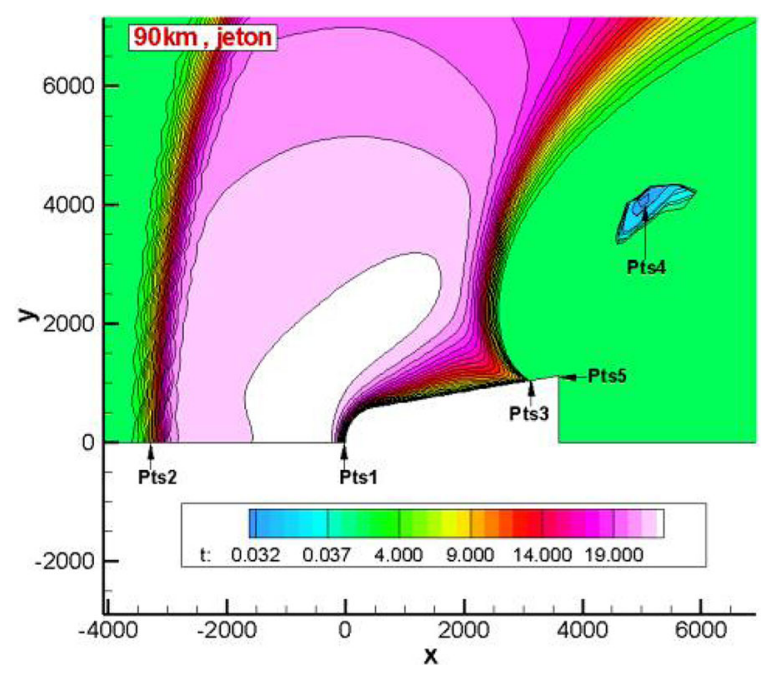

Fig. 1 Temperature contour for the jet case

where the subscripts $(\mathrm{i} 1, \mathrm{j} 1, \mathrm{k} 1)$ indicates the cell sharing the iith edge with the $(\mathrm{i}, \mathrm{j}, \mathrm{k})$ cell. The quantity $F F$ can be expressed as

$$
\begin{aligned}
& F F\left((\Delta f)_{i, j, k, l, m, n},(\Delta f)_{i 1, j 1, k 1, l, m, n}\right) \\
& \quad=\frac{1}{2}\left[(\Delta f)_{i, j, k, l, m, n}+(\Delta f)_{i 1, j 1, k 1, l, m, n}\right]+\frac{1}{2} \operatorname{sign}\left(\mathbf{u}_{l, m, n} \cdot \mathbf{n}_{i i}\right) \\
& \quad\left[(\Delta f)_{i, j, k, l, m, n}-(\Delta f)_{i 1, j 1, k 1, l, m, n}\right]
\end{aligned}
$$

Substituting the above expression into Eq. (15) we can get

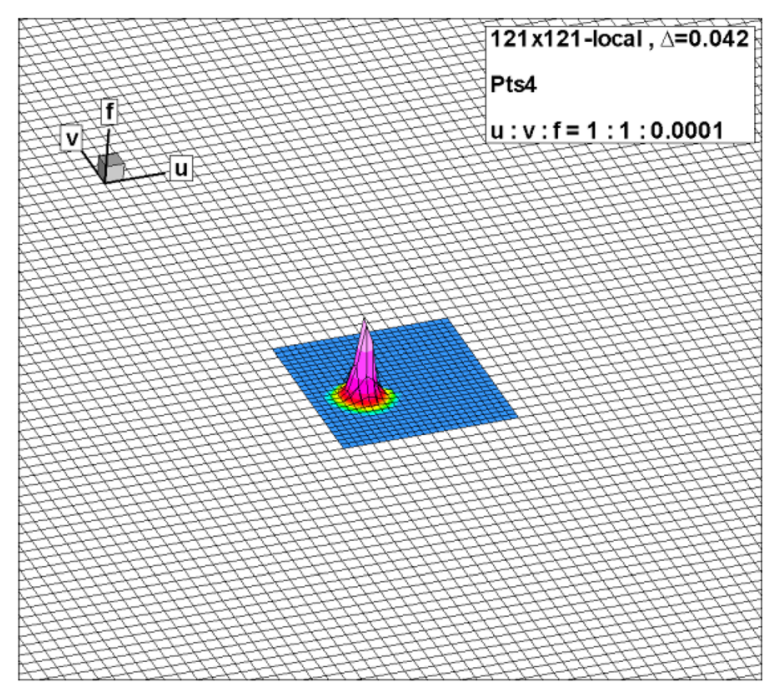

Fig. 2 Distribution function at pts4 


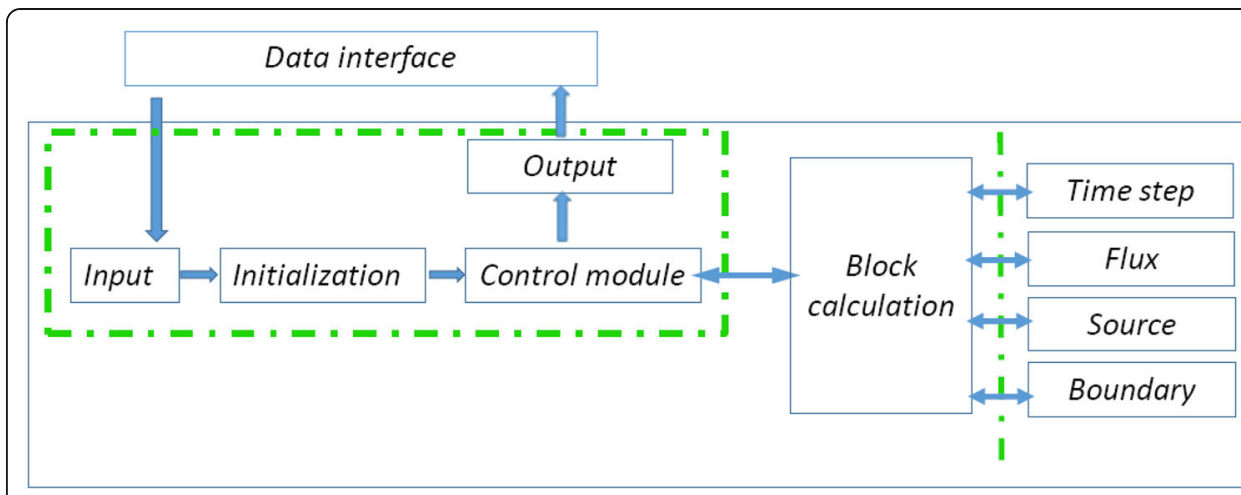

Fig. 3 Framework of UGKS solver package

$$
\begin{aligned}
& {\left[1+\Delta t \cdot \frac{1}{\tau^{\zeta}}+\Delta t \cdot b_{i, j, k, l, m, n}\right](\Delta f)_{i, j, k, l, m, n}+\sum_{i i=1}^{6} \Delta t \cdot c_{i, j, k, l, m, n} \cdot(\Delta f)_{i 1, j 1, k 1, l, m, n}=\Delta t \cdot R_{i, j, k, l, m, n}^{\zeta}} \\
& b_{i, j, k, l, m, n}=\sum_{i i=1}^{6}\left(\mathbf{u}_{l, m, n} \cdot \mathbf{n}_{i i}\right) \cdot\left(1+\operatorname{sign}\left(\mathbf{u}_{l, m, n} \cdot \mathbf{n}_{i i}\right)\right) \frac{\left|S_{i, j, k, i i}\right|}{2\left|V_{i, j, k}\right|} \\
& c_{i, j, k, l, m, n}=\left(\mathbf{u}_{l, m, n} \cdot \mathbf{n}_{i i}\right) \cdot\left(1-\operatorname{sign}\left(\mathbf{u}_{l, m, n} \cdot \mathbf{n}_{i i}\right)\right) \frac{\left|S_{i, j, k, i i}\right|}{2\left|V_{i, j, k}\right|}
\end{aligned}
$$

Writing Eq. (16) in matrix form

$$
\begin{gathered}
\left(\mathbf{I}+\Delta t \cdot \mathbf{Z}_{l, m, n}\right) \cdot(\Delta \mathbf{f})_{l, m, n}=\Delta t \cdot \mathbf{X}_{l, m, n}^{-1} \cdot \mathbf{R}_{l, m, n}^{\zeta} \\
(\Delta \mathbf{f})_{l, m, n}=\left(\begin{array}{l}
(\Delta f)_{1,1,1, l, m, n} \\
(\Delta f)_{2,1,1, l, m, n} \\
\cdots \\
(\Delta f)_{N I-1, N J-1, N K-1, l, m, n}
\end{array}\right) \\
\mathbf{R}_{l, m, n}^{\zeta}=\left(\begin{array}{l}
R_{1,1,1, l, m, n}^{\zeta} \\
R_{2,1,1, l, m, n}^{\zeta} \\
\cdots \\
R_{N I-1, N J-1, N K-1, l, m, n}^{\zeta}
\end{array}\right) \\
\mathbf{X}_{l, m, n}=\left(\begin{array}{llll}
X_{1,1,1, l, m, n} & 0 & \cdots & 0 \\
0 & X_{2,1,1, l, m, n} & \cdots & 0 \\
0 & 0 & \cdots & 0 \\
0 & 0 & \cdots & X_{N I-1, N J-1, N K-1, l, m, n}
\end{array}\right)
\end{gathered}
$$

Table 1 Free-stream conditions

\begin{tabular}{lllllllll}
\hline Configuration & Mach & $\mathrm{Kn}$ & $\lambda$ definition & Working gas & $\bar{L}(\mathrm{~m})$ & $\omega$ & $T_{\infty}(\mathrm{K})$ & $T_{w}(\mathrm{~K})$ \\
\hline Wedge & 10 & 0.05 & $\mathrm{HS}$ & Argon & 0.2 & 0.81 & 200 & 300 \\
Cylinder & 1.96 & $0.0162,0.162$ & VHS & Nitrogen & radius & 0.74 & 124.94 & 259.87 \\
Cylinder & 5 & $0.01,0.1,1$ & VHS & Argon & radius & 0.81 & 273 & 273 \\
Cylinder & 10 & $3.03 \mathrm{e}-3,7.58 \mathrm{e}-2$ & VHS & Argon & radius & 0.81 & 200 & 500 \\
Cylinder & 25 & $3.69 \mathrm{e}-3,1.84 \mathrm{e}-2$ & VHS & Argon & radius & 0.734 & 200 & 1500 \\
& & $9.22 \mathrm{e}-2,0.461$ & & & & & & \\
Cone & 8.1 & $9.75 \mathrm{e}-3,3.38 \mathrm{e}-1$ & VHS & Argon & 0.02 & 0.81 & 247,189 & 273 \\
Sphere & 4.25 & $0.031 \sim 0.672$ & VHS & Nitrogen & 0.002 & 0.74 & 65 & 302 \\
Sphere & 5.45 & $0.256 \sim 1.96$ & VHS & Nitrogen & 0.002 & 0.74 & 43 & 315 \\
X38-like & 4 & $8.41 \mathrm{e}-5 \sim 8.41 \mathrm{e}-2$ & VHS & Argon & 0.28 & 0.81 & 56 & 300 \\
X38-like & 6 & $1.26 \mathrm{e}-4 \sim 1.26 \mathrm{e}-1$ & VHS & Argon & 0.28 & 0.81 & 56 & 300 \\
X38-like & 8 & $1.68 \mathrm{e}-4 \sim 1.68 \mathrm{e}-1$ & VHS & Argon & 0.28 & 0.81 & 56 & 300 \\
\hline
\end{tabular}




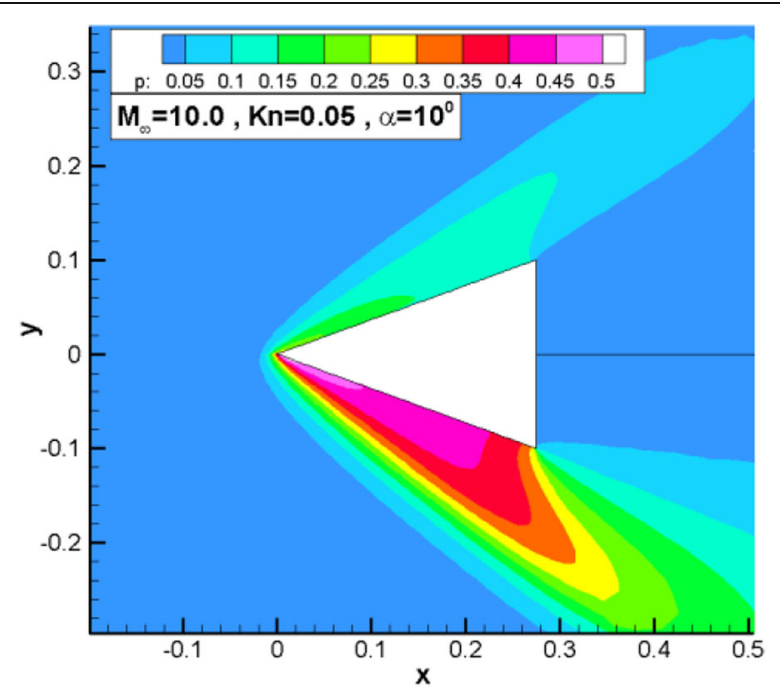

Fig. 4 Pressure contour of the wedge

where NI, NJ and $N K$ are the physical mesh points in the $i, j$ and $k$ directions, respectively.

Applying approximate $\mathrm{LU}$ decomposition to $\left(\boldsymbol{I}+\Delta t \cdot \boldsymbol{Z}_{l, m, n}\right)$ we can get

$$
\mathbf{I}+\Delta t \cdot \mathbf{Z}_{l, m, n}=\mathbf{L}_{l, m, n} \cdot \mathbf{U}_{l, m, n}+\bigcirc\left(\Delta t^{2}\right)
$$

Where $\mathbf{L}_{l, m, n}$ and $\mathbf{U}_{l, m, n}$ are both diagonal matrices and can be given by

$$
l_{p q}=\left\{\begin{array}{ll}
\Delta t \cdot z_{p q} & p<q \\
0 & p>q
\end{array} u_{p q}=\left\{\begin{array}{ll}
0 & p<q \\
\Delta t \cdot z_{p q} & p>q
\end{array} l_{p p}=u_{p p}=1\right.\right.
$$

The implicit method in the final form reads

$$
\mathbf{L}_{l, m, n} \cdot \mathbf{U}_{l, m, n} \cdot(\Delta \mathbf{f})_{l, m, n}=\Delta t \cdot \mathbf{X}_{l, m, n}^{-1} \cdot \mathbf{R}_{l, m, n}^{\zeta}
$$

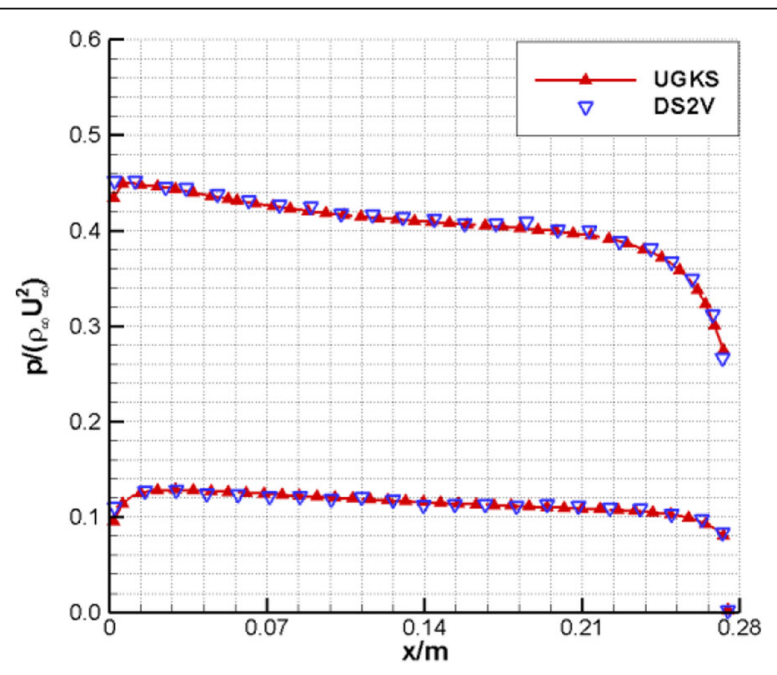

Fig. 5 Pressure distribution on the wedge surface 


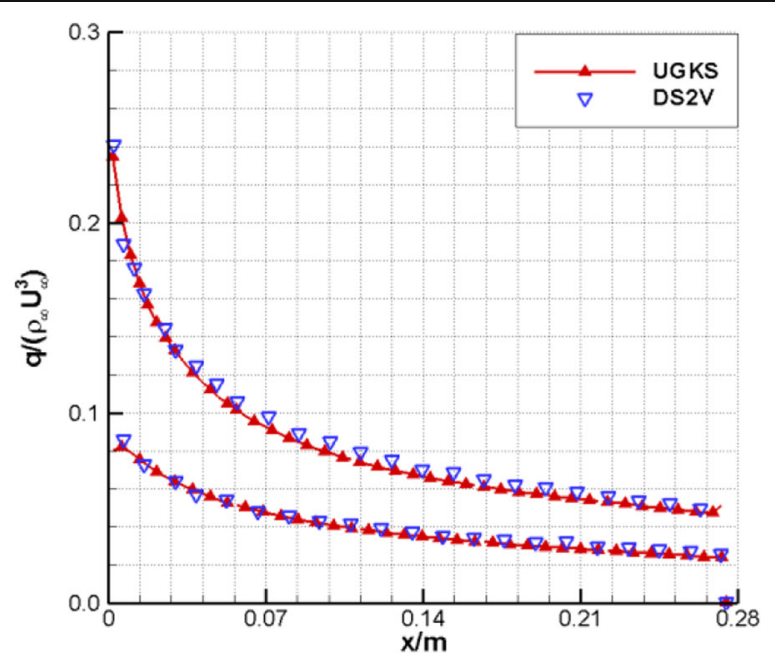

Fig. 6 Heat flux distribution on the wedge surface

In structured meshes, $(\Delta \mathbf{f})_{l, m, n}$ can be obtained after backward and forward substitution and $f^{\zeta+1}$ can be got subsequently.

In the above procedure, the gain term $f^{+}$in the collision term is treated explicitly. Since UGKS is a multi-scale hybrid method with both macroscopic and microscopic variable updates. The macroscopic variables can be updated implicitly first to give a pre-evaluating $f^{+}$, resulting in a complete implicit implementation [26] for the collision term. This is very useful for continuum or near continuum flows.

\subsection{Local refinement in the velocity mesh}

Generic adaptive mesh refinement (AMR) $[27,28]$ in velocity can greatly decrease the $\mathrm{CPU}$ time and memory requirements for UGKS. However, the resulting velocity meshes are usually different for different spatial cells, making it rather difficult to apply the implicit technique.

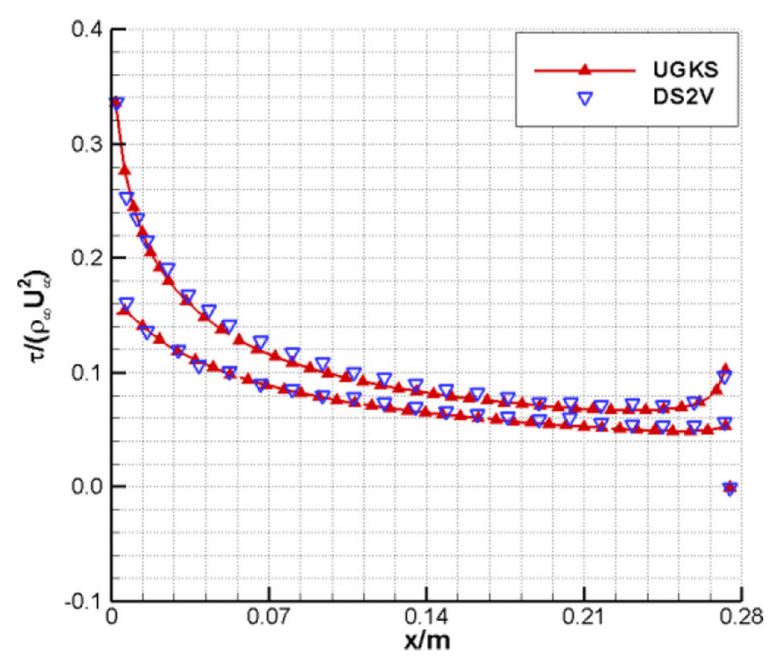

Fig. 7 Shear stress distribution on the wedge surface 


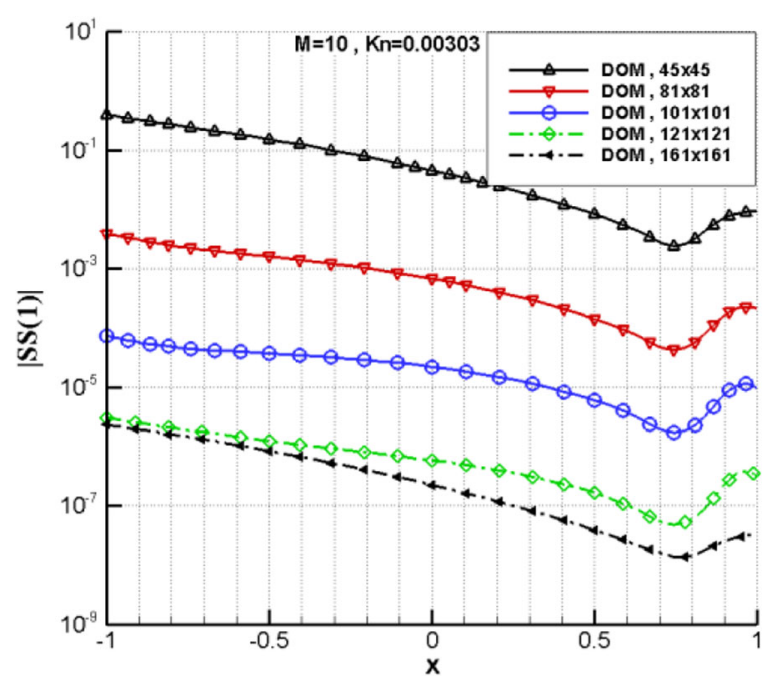

Fig. $8|S S(1)|$ on the cylinder surface

In our UGKS solver package, we combine the merits of both methods through the following procedure. First, the bounds and interval of a global uniform velocity mesh are calculated according to numerical experiences or a pre-conducted Navier-Stokes simulation results. Obviously, the lower and upper limits of the velocity mesh in each direction are determined by the highest temperature which usually appears in the shock layer. While the mesh interval $\Delta v$ is determined by the lowest temperature in the whole field. Second, a global uniform velocity mesh is generated which we call background mesh. The interval of this mesh is $a \cdot \Delta v$ where $a$ is larger than one. Then we give a patch on the background velocity mesh for the spatial cells whose velocity mesh interval should be less than $a \cdot \Delta v$. The location of the patch can be determined by the pre-calculated Navier-Stokes results or even by the UGKS results with the background velocity mesh. The resulting velocity mesh is still structured. The implicit method can be applied without any difficulties.

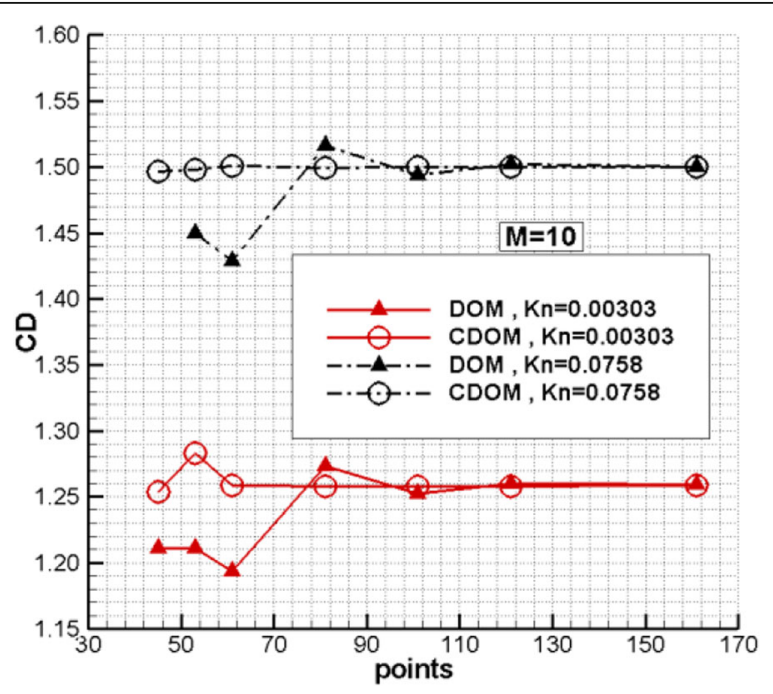

Fig. 9 Cylinder drag coefficient vs points in $u(v)$ direction 


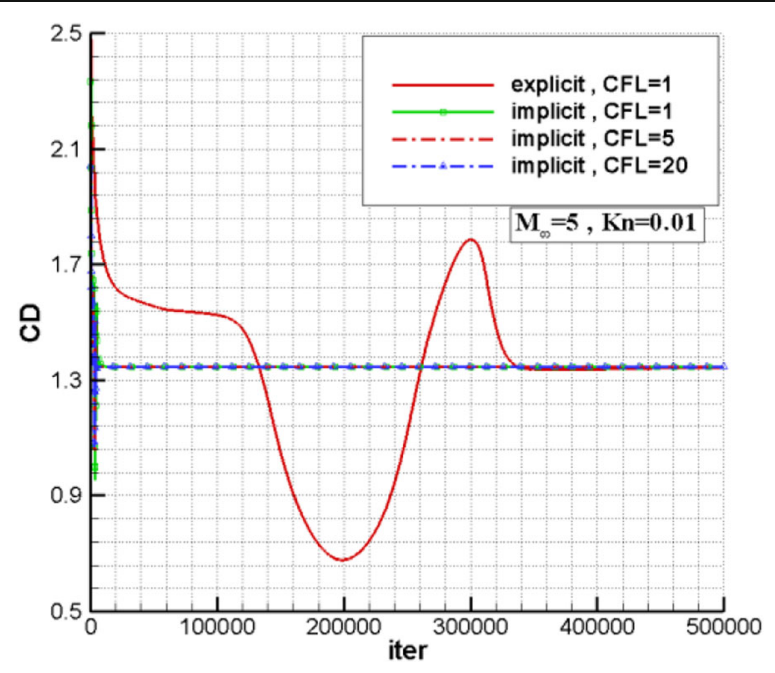

Fig. 10 Convergent histories of the drag coefficient with $\mathrm{Ma}=5.0$ and $\mathrm{Kn}=0.01$

Up to now, the only difficulty arising may be the interpolation of distribution functions from the background mesh to the patch. We use the following conservative method. Take 1D case for example, the composite Newton-Cote's quadrature requires that the total number of velocity points is $4 \mathrm{~N}+1$, where $\mathrm{N}$ is a positive integer. We can get an interpolation polynomial from the five distribution functions which is equally spaced on a small block of four successive intervals on the velocity mesh. Since Newton-Cote's quadrature coefficients are derived from this polynomial, they are consistent. It can be easily proved that the conservations of mass, momentum and energy hold if we extend the original 5 points equally spaced mesh to a 9 points equally spaced mesh. For $2 \mathrm{D}$ or $3 \mathrm{D}$ cases, extending a block mesh of $5 \times 5$ or $5 \times 5 \times 5$ to $9 \times 9$ or $9 \times 9 \times$ 9 can be done in the same way. Proof of the conservation law can be verificated through some mathematical software such as MAPLE.

We have applied this technique in a 2D jet case on a blunt cone. The freestream Mach number is 8.1 with an altitude of $90 \mathrm{~km}$. The jet condition is $\rho_{j}=7.468 e-3$,

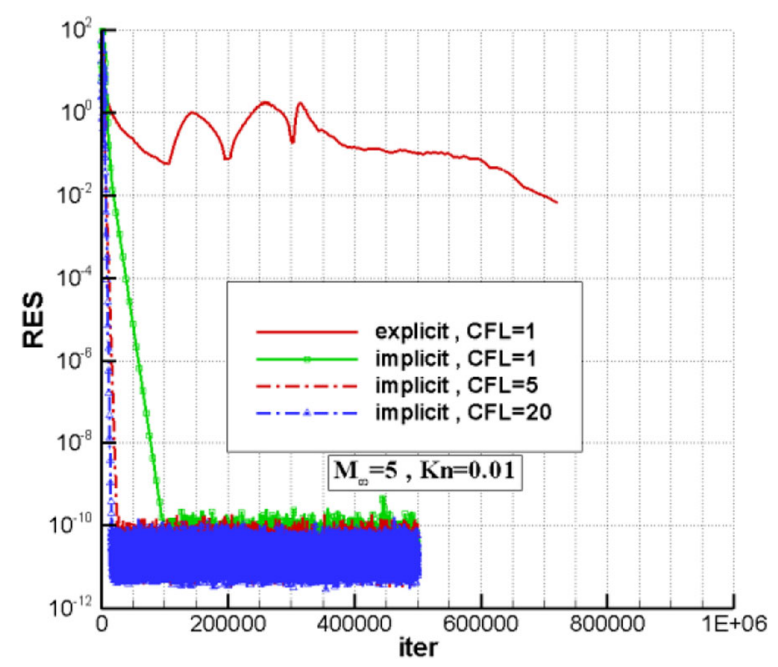

Fig. 11 Convergent histories of the residual with $\mathrm{Ma}=5.0$ and $\mathrm{Kn}=0.01$ 
Table 2 Comparison of the explicit and implicit methods in convergence rate

\begin{tabular}{lllll}
\hline $\mathrm{Ma}$ & $\mathrm{Kn}$ & Nc.E & Nc.l & Rs = Nc.E/ Nc.I/1.02 \\
\hline 5 & 0.01 & 502,450 & 5800 & 84.93 \\
5 & 0.1 & 463,500 & 3500 & 129.83 \\
10 & 0.01 & 505,900 & 4645 & 106.78 \\
\hline
\end{tabular}

$u_{j}=c_{j}, \mathrm{p}_{j}=373 \mathrm{~Pa}, \mathrm{~T}_{j}=240 \mathrm{~K}$. The pressure ratio of the jet to the free-stream is about 2000. For the jet-off case, a velocity mesh of $121 \times 121$ is enough. For the jet-on case, the local temperature decreases severely due to rapid expansion from the jet exit. Figure 1 shows the temperature contour. The temperature in the downstream of the jet near pts4 is about one order lower than the free-stream temperature. Thus, it's necessary to refine the velocity mesh in order to resolve the corresponding distribution function. From the pre-conducted UGKS results, we choose 9 blocks of $5 \times 5$ sub-mesh and extend them to $9 \times 9$ sub-mesh. The final distribution function and the velocity mesh are shown in Fig. 2.

In this case, if we use global uniform mesh, the total mesh will be $241 \times 241$. With the local refinement technique, the total mesh is $121 \times 121+9 \times(9 \times 9-5 \times 5)=15,145$ which is only $1 / 3.8$ of the former.

\subsection{Parallelization}

At present, hybrid parallelization similar to that in [16] is used. The space mesh is decomposed and parallelized with MPI which has been broadly applied in many traditional CFD software. In every MPI process, several threads are used with OpenMP. However, due to the architecture change of our new super cluster, three space dimensions and one velocity dimension decomposition technique is under developing, allowing for a larger parallel scale up to 10,000 cores in the near future.

\section{Code framework}

The UGKS solver package is based on the framework of our in-house NS solver, CARDC Hypersonic Aerodynamic Numerical Tunnel (CHANT) [29]. Figure 3 shows

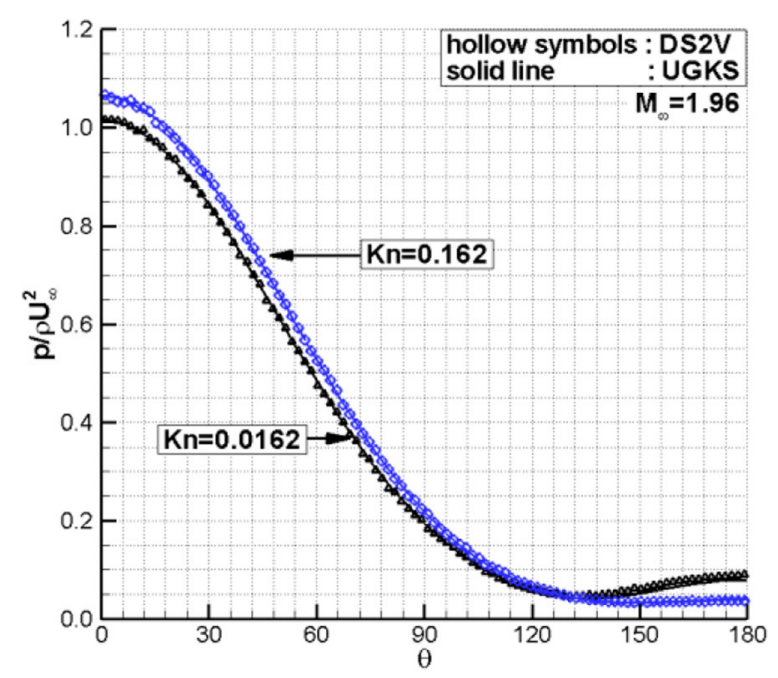

Fig. 12 Pressure distributions on the cylinder surface at $M=1.96$ 


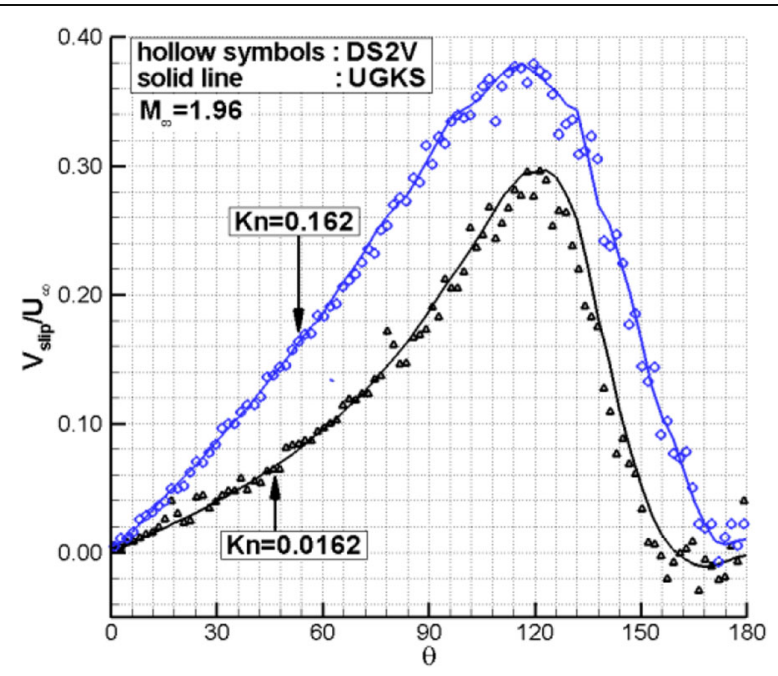

Fig. 13 Slip velocity distributions on the cylinder surface at $M=1.96$

the general sketch. The whole package is composed of five parts: input, output, initialization, control and calculation. The flowfield of a certain configuration is obtained through calculations over all structured blocks one by one. Multi-stage interface is devised for further development. Fortran90 is used for all subroutines.

The current features of UGKS solver package can be summarized as follows:

- 2D and 3D body-fitted structured multi-block mesh

- Steady and unsteady simulations

- Explicit and implicit methods

- Conservative discrete ordinate method

- Local refinement in velocity mesh

- Shakhov model for monatomic gases

- Rykov model for diatomic gases

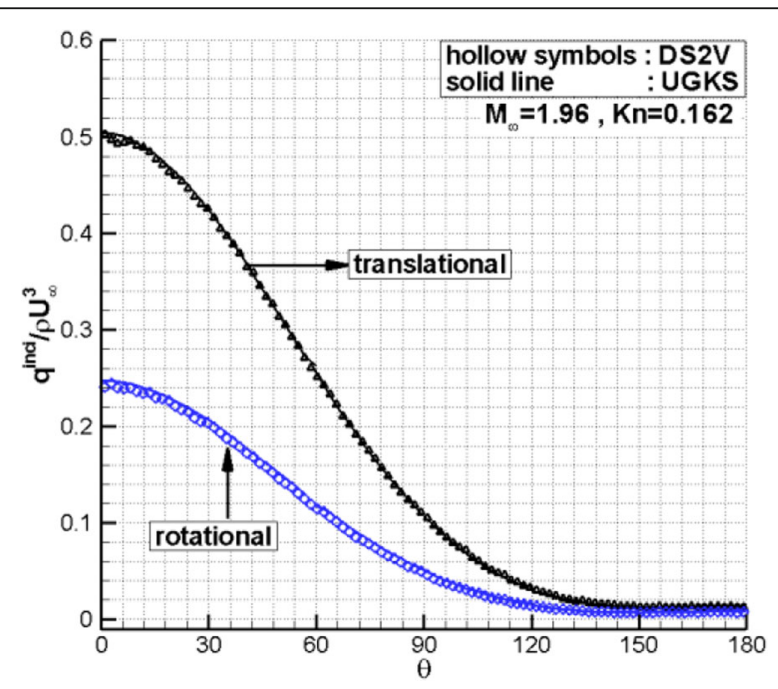

Fig. 14 Incident heat flux distributions on the cylinder surface at $M=1.96$ 


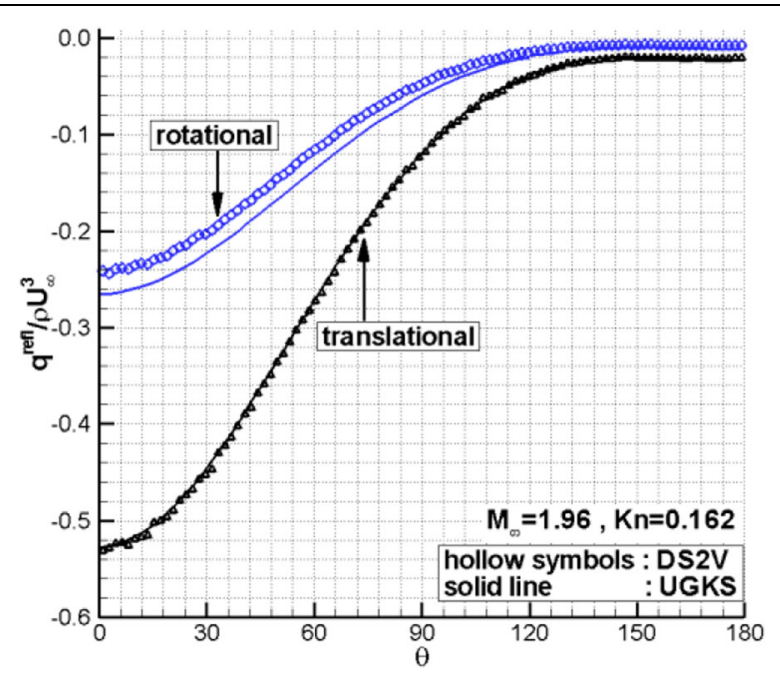

Fig. 15 Reflective heat flux distributions on the cylinder surface at $M=1.96$

- Diffuse or specular reflection wall boundaries, free-stream boundary, outflow boundary, symmetrical boundary

- Several models for the viscosity calculation such as hard sphere model, variable hard sphere model [30] or the Sutherland model

- Hybrid parallelization with MPI and OpenMP

\section{Validation cases}

Five test cases are considered. UGKS results are compared with those obtained from either DS2V [5], MONACO [31], RariHV [32] or experiments. Fully diffuse solid boundary is used. In all cases, the global Knudsen number Kn is defined as

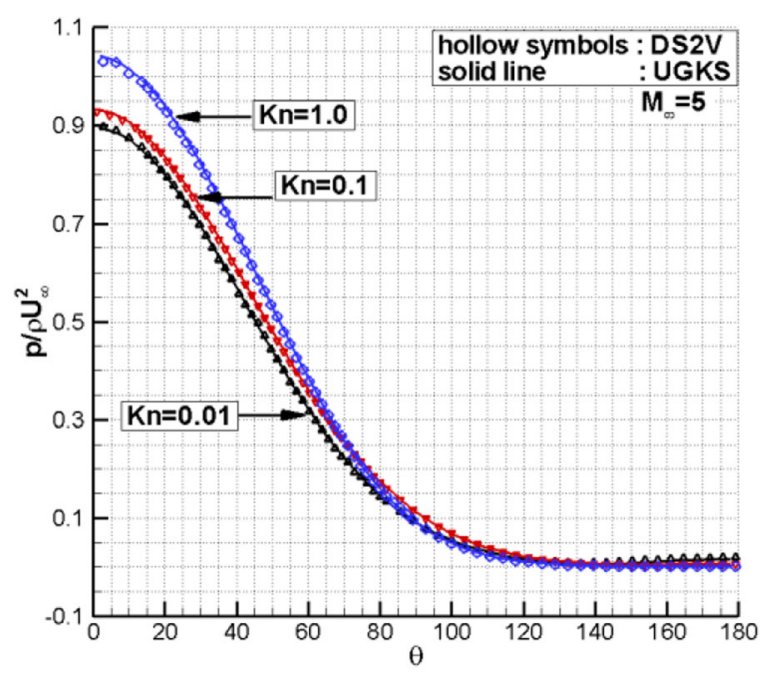

Fig. 16 Pressure distributions on the cylinder surface at $M=5$ 


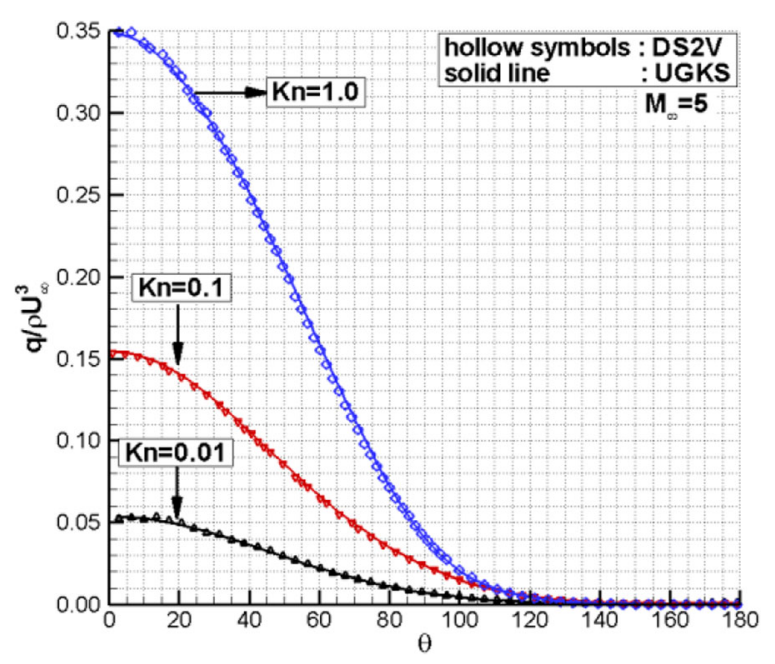

Fig. 17 Heat flux distributions on the cylinder surface at $M=5$

$$
K n=\frac{\lambda}{\bar{L}}
$$

where $\lambda$ is the mean free path which is determined for either hard sphere (HS) molecules [30].

$$
\lambda=\frac{16}{5} \sqrt{\frac{m}{2 \pi k T}} \frac{\mu}{\rho}
$$

or variable hard sphere (VHS) molecules

$$
\lambda=\frac{2(5-2 \omega)(7-2 \omega)}{15} \sqrt{\frac{m}{2 \pi k T}} \frac{\mu}{\rho}
$$

where $\omega$ is the power law index of the viscosity, $m$ is the atomic mass, $k$ is the Boltzmann constant.

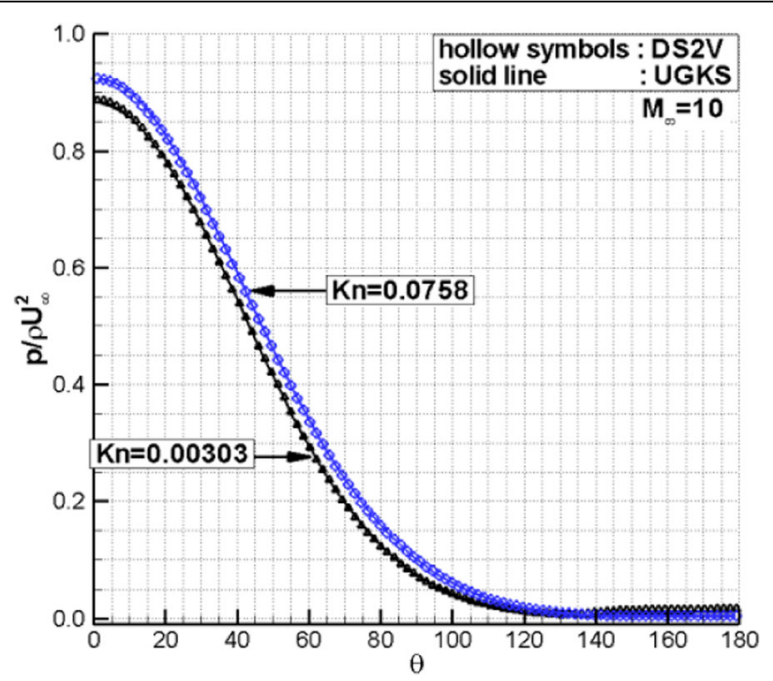

Fig. 18 Pressure distributions on the cylinder surface at $M=10$ 


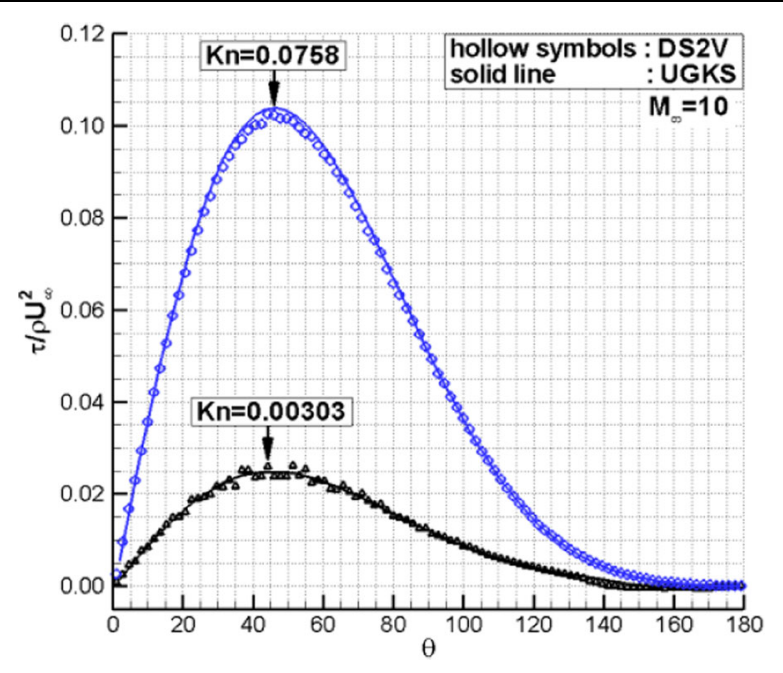

Fig. 19 Shear stress distributions on the cylinder surface at $M=10$

The main free-stream conditions for all cases are summarized in Table 1.

\subsection{Hypersonic flow over a $40^{\circ}$ wedge}

The angle of attack is 10 degrees. Figure 4 shows the pressure contour predicted by UGKS. Figures 5, 6 and 7 display the pressure, heat flux and shear stress distributions on the surface, respectively. The UGKS results and DS2V results are almost identical, indicating that UGKS code package and DS2V can predict flows with similar accuracy.

\subsection{Super and hypersonic flows over a 2D cylinder}

This is a quite comprehensive test case covering supersonic and hypersonic flows in all regimes. We also use this case for validating the CDOM and implicit techniques described in section 2 .

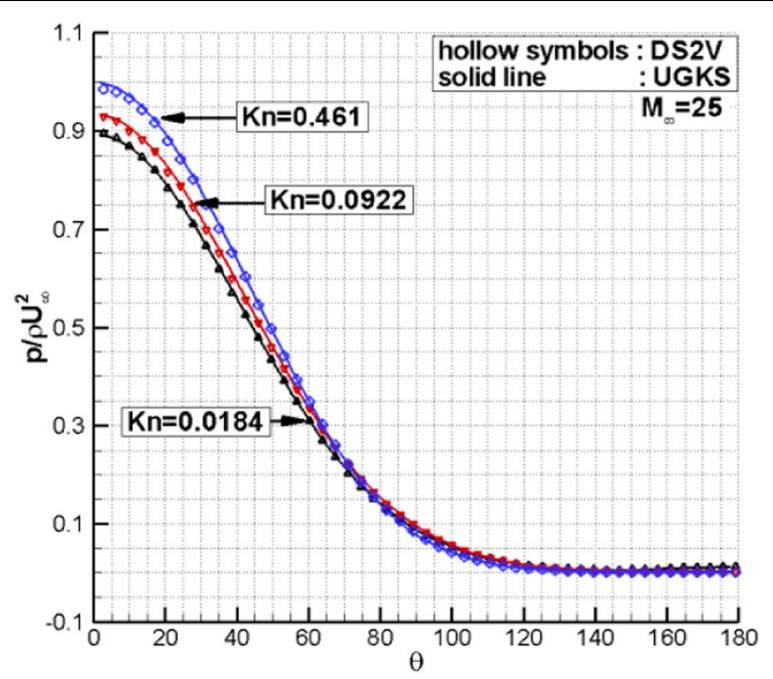

Fig. 20 Pressure distributions on the cylinder surface at $M=25$ 


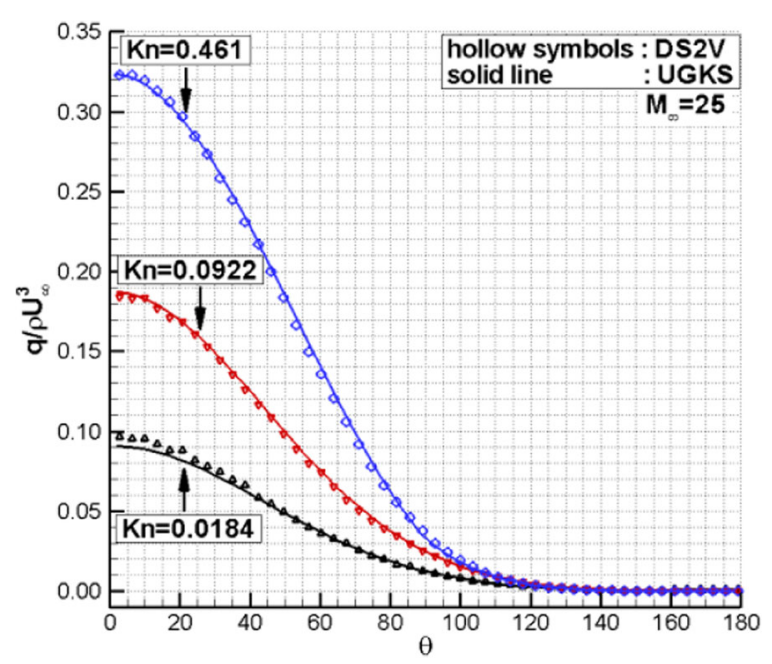

Fig. 21 Heat flux distributions on the cylinder surface at $M=25$

For Mach number 10, both DOM and CDOM calculations are conducted. Figure 8 shows the variable $|\mathbf{S S}(1)|$ in the cells just near the wall at different velocity space meshes. When the velocity space mesh increases, the numerical source term decreases but will stay at a certain level finally. So increasing the velocity space mesh will not eliminate the source term. However, the source term will be on an order of $10^{-14} \sim 10^{-15}$ if CDOM is applied. The total drag at different velocity space meshes is given in Fig. 9. Obviously, the mesh dependence with CDOM is much smaller than that with DOM. The solution at $61 \times 61$ mesh with $\mathrm{CDOM}$ can be considered as mesh convergent while with DOM the same result can only be obtained at a much finer mesh of $121 \times 121$. Thus, the time and memory cost will decrease by nearly three quarters with the help of CDOM.

Figures 10 and 11 show the convergent histories of the drag coefficient and residual for Mach number 5 and Knudsen number 0.01, respectively. A comparison of the explicit and implicit methods in convergence rate is shown in Table 2. Nc.E and Nc.I are the total iterations steps for a convergent solution for the explicit and implicit methods,

Table 3 Comparisons of cylinder drag

\begin{tabular}{lllll}
\hline $\mathrm{M}_{\infty}$ & $\mathrm{Kn}_{\infty}$ & UGKS & DS2V/MONACO & Relative error (\%) \\
\hline 1.96 & 0.0162 & 1.597 & 1.582 & 0.92 \\
1.96 & 0.162 & 1.862 & 1.863 & -0.06 \\
5 & 0.01 & 1.320 & 1.316 & 0.31 \\
5 & 0.1 & 1.527 & 1.523 & 0.28 \\
5 & 1 & 1.929 & 1.917 & 0.62 \\
5.43 & 0.303 & 1.774 & 1.775 & -0.05 \\
5.43 & 1.52 & 2.277 & 2.304 & -1.19 \\
10 & 0.00303 & 1.258 & 1.252 & 0.56 \\
10 & 0.0758 & 1.500 & 1.496 & 0.27 \\
25 & 0.00369 & 1.256 & $\mathrm{~N}-\mathrm{A} / 1.259$ & -0.21 \\
25 & 0.0184 & 1.348 & $1.349 / 1.347$ & 0.07 \\
25 & 0.0922 & 1.531 & $1.521 / 1.528$ & 0.22 \\
25 & 0.461 & 1.807 & $1.792 / 1.771$ & 2.03 \\
\hline
\end{tabular}




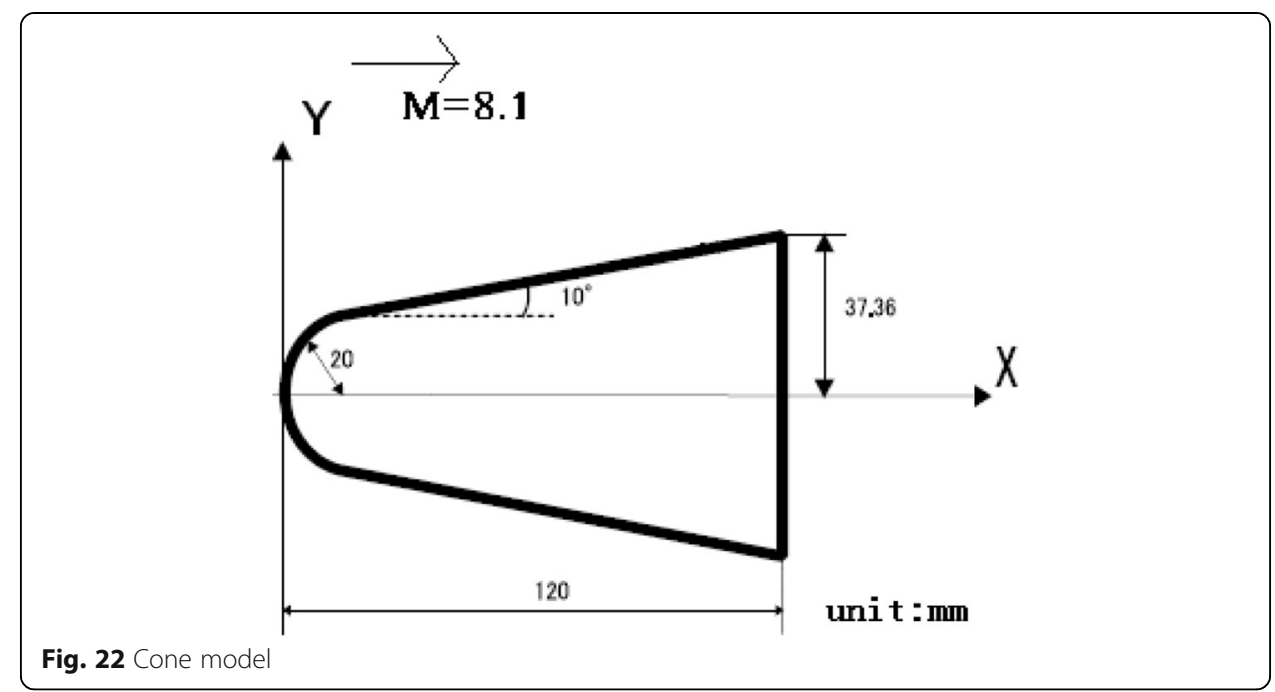

respectively. Rs is the speed-up ratio for the implicit method, where the denominator 1.02 comes from the fact that the computational cost of one time step for the implicit method is about $2 \%$ more than that for the explicit method. A speed up ratio of nearly two orders can be achieved.

Figures 12, 13, 14 and 15 show the comparisons between UGKS and DS2V for a diatomic nitrogen gas. The UGKS results are obtained with the Rykov model with rotational degrees of freedom. Thus, the heat flux can be divided into two parts, the contributions of translational degree and rotational degree. Good agreements can be seen, providing a sound validation for our UGKS code for diatomic gases.

Figures 16 and 17 are the results for Mach number 5. Figures 18 and 19 are the results for Mach number 10. Figures 20 and 21 are the results for Mach number 25. We omit some comparisons at certain Mach numbers because of space limitations.

Table 3 gives the drag coefficient comparisons. The maximum relative error is only 2.03\%.

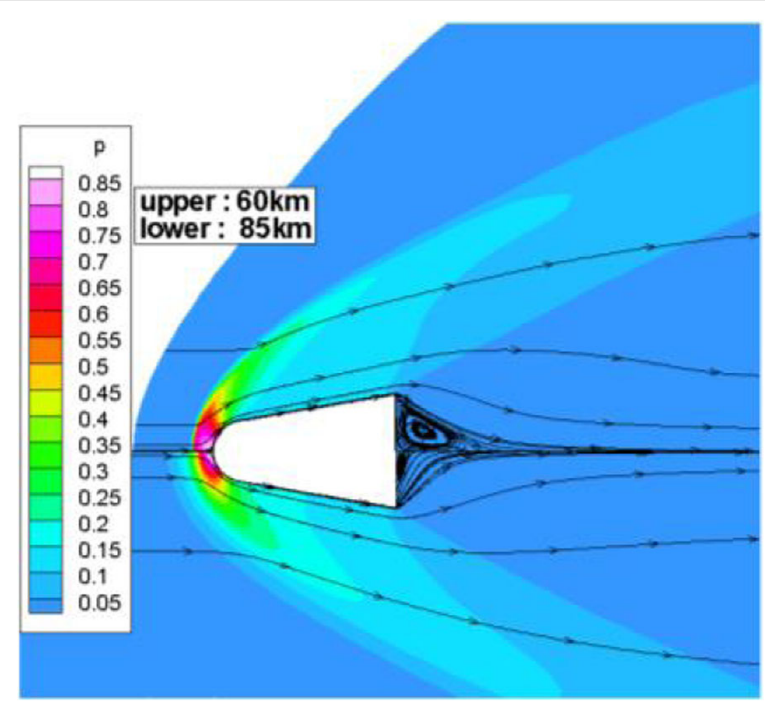

Fig. 23 Pressure contour and streamlines 


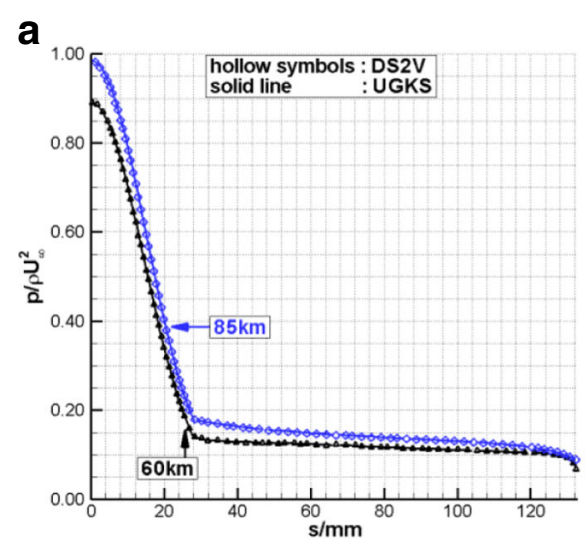

Body

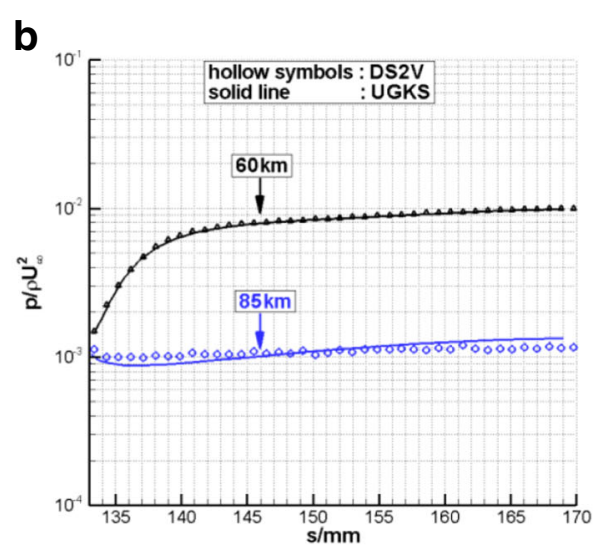

Bottom

Fig. 24 Pressure distributions on the cone surface (a) Body (b) Bottom

\subsection{Hypersonic flow over a 2D cone}

Figure 22 gives the computational configuration. The angle of attack is 0 degree. The pressure contour and streamlines are shown in Fig. 23. The altitude in the figure is only 'nominal' which means that only the temperature and number density at the corresponding altitude are used, since the air is treated as a monatomic gas. In other words, internal degrees of freedom are ignored. The two global Knudsen numbers in Table 1 for cone case correspond to nominal altitudes $60 \mathrm{~km}$ and $85 \mathrm{~km}$, respectively. The flow pattern is relatively simple, i.e., a bow shock in front of the blunt body and a vortex in the bottom similar to that in a backward step case. However, the bow shock in front of the $85 \mathrm{~km}$ case is much weaker than that in the $60 \mathrm{~km}$ case. The recirculation zone in the bottom is smaller, too.

Figures 24, 25 and 26 show the pressure, heat flux, and shear stress distributions on the cone surface, respectively. The abscissa indicates the distance from the very begin of the cone on the surface. The bottom pressure at $60 \mathrm{~km}$ rises about one order from the corner to the center of the bottom, resulting in a large adverse pressure gradient and inducing a large separation. At $85 \mathrm{~km}$, the pressure curve is rather flat and only
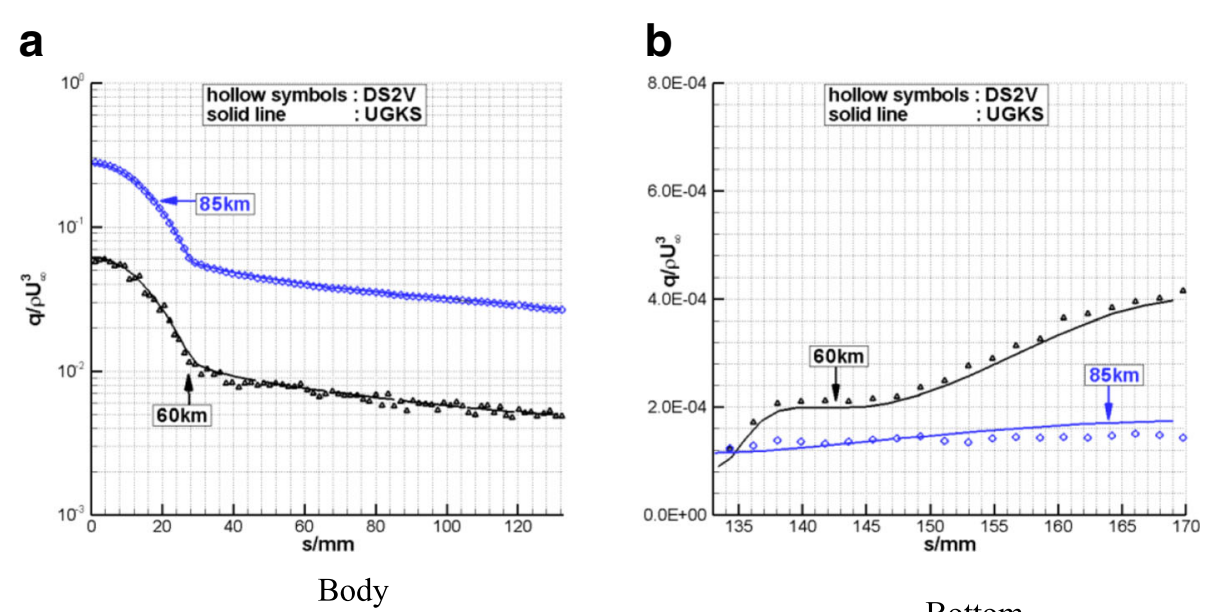

Fig. 25 Heat flux distributions on the cone surface (a) Body (b) Bottom

\section{Bottom}



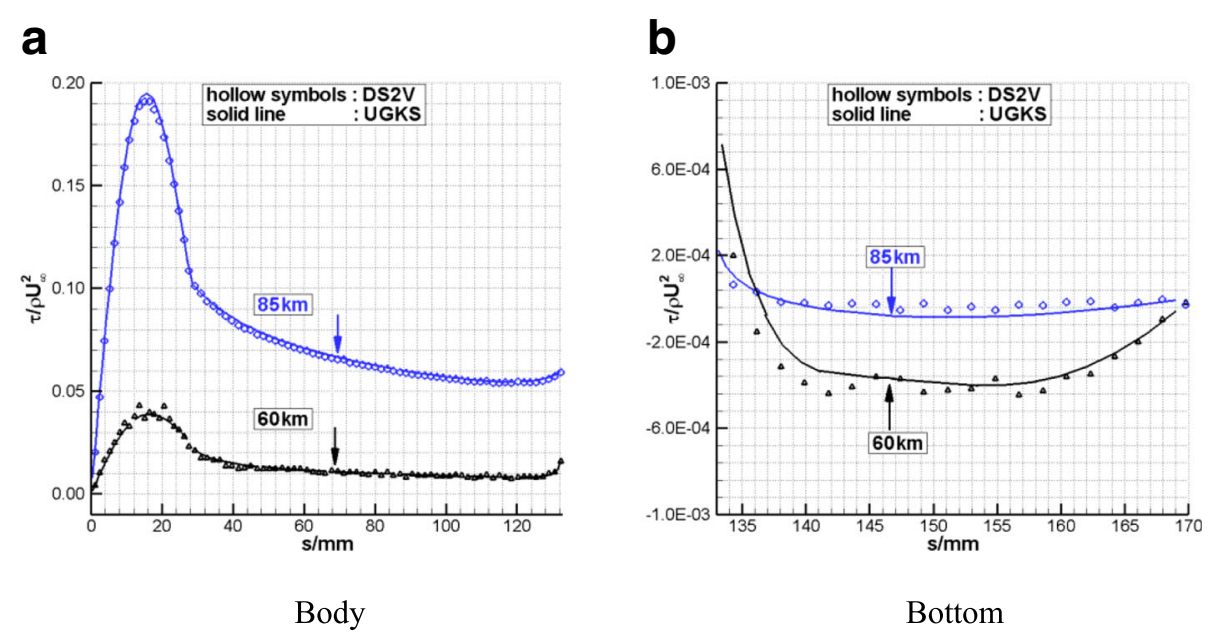

Fig. 26 Shear stress distributions on the cone surface (a) Body (b) Bottom

small adverse pressure gradient occurs. Moreover, the minimum pressure, heat flux and stress at the bottom are almost three orders lower than the maximum values on the cone. UGKS can capture these phenomena as accurately as the DS2V.

\subsection{Supersonic and hypersonic flows over a sphere}

The flow past a sphere is simulated with Rykov model to compare with the experimental drag coefficients [33]. The space mesh contains 21,840 cells while a velocity mesh of $41 \times 41 \times 41$ is used.

Figure 27 shows the pressure contour for two cases. When the Knudsen number is large, variable gradient in the whole field is small. There is only weak compressive wave in front of the sphere.

a

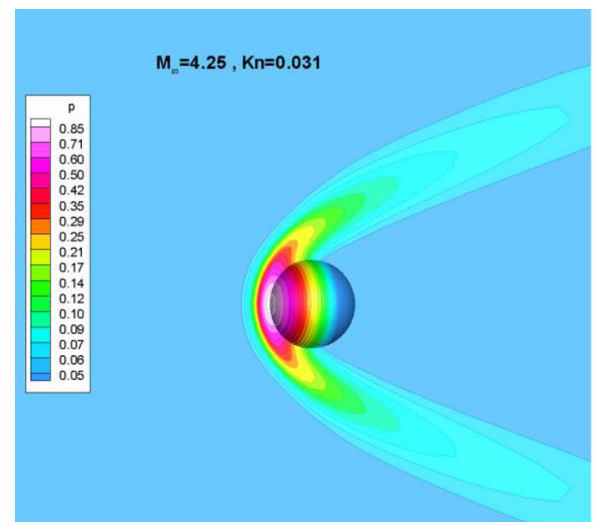

$\mathrm{M}=4.25, \mathrm{Kn}=0.031$ b

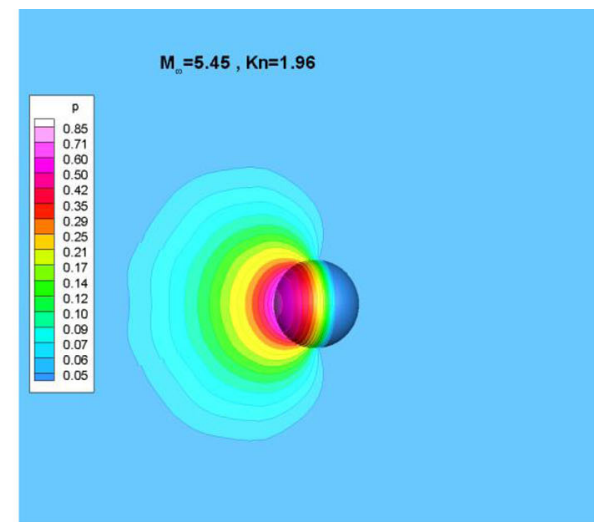

$\mathrm{M}=5.45, \mathrm{Kn}=1.96$

Fig. 27 Pressure contour on the symmetry plane and the sphere surface (a) $M=4.25, K n=0.031$ (b) $M=5.45, K n=1.96$ 
Table 4 Comparisons of sphere drag

\begin{tabular}{llllll}
\hline$M_{\infty}$ & $\mathrm{Re}$ & $\mathrm{Kn}_{\infty}$ & UGKS (Nitrogen) & Exp (Air) & Relative error (\%) \\
\hline 4.25 & 9.55 & 0.672 & 2.356 & 2.42 & $2.64 \%$ \\
4.25 & 19.0 & 0.338 & 2.101 & 2.12 & $0.87 \%$ \\
4.25 & 53.0 & 0.121 & 1.694 & 1.69 & $-0.27 \%$ \\
4.25 & 80.5 & 0.080 & 1.558 & 1.53 & $-1.80 \%$ \\
4.25 & 150.0 & 0.043 & 1.410 & 1.37 & $-2.91 \%$ \\
4.25 & 210.0 & 0.031 & 1.355 & 1.35 & $-0.39 \%$ \\
5.45 & 4.2 & 1.960 & 2.595 & 2.60 & $0.18 \%$ \\
5.45 & 8.6 & 0.957 & 2.449 & 2.44 & $-0.36 \%$ \\
5.45 & 16.8 & 0.490 & 2.248 & 2.28 & $1.41 \%$ \\
5.45 & 32.1 & 0.256 & 2.005 & 2.04 & $1.71 \%$ \\
\hline
\end{tabular}

Table 4 gives the drag coefficient comparisons. The maximum relative error is only 2.64\%. The agreements can be considered as excellent since the root mean square (RMS) error of the experiments is about $\pm 2 \%$.

\subsection{Supersonic and hypersonic flows over a X38-like vehicle}

The angle of attack is 20 degrees in this case. The space mesh contains 334,434 cells while a velocity mesh of $33 \times 33 \times 33$ is used. The total six-dimensional mesh reaches $1.2 \times 10^{10}$. The reference area for the aerodynamic coefficient is $2.41 \times 10^{-2} \mathrm{~m}^{2}$.

Figure 28 gives the spatial streamlines around the vehicle with Mach number 4 . When the free-stream Knudsen number is relatively small, the adverse pressure gradient can be large enough to induce the flow to separate from the boundary, resulting in the vortex in Fig. 28(a).

Figure 29 shows the local Knudsen number distribution near the surface. Local Knudsen number is calculated through Eq. (19) with the characteristic length $\bar{L}$ substituted by the local gradient-length $Q /|d Q / d l|$ proposed by Boyd [34]. In this paper, the density-based gradient-length is used. The local Knudsen number can cover a wide range of values with

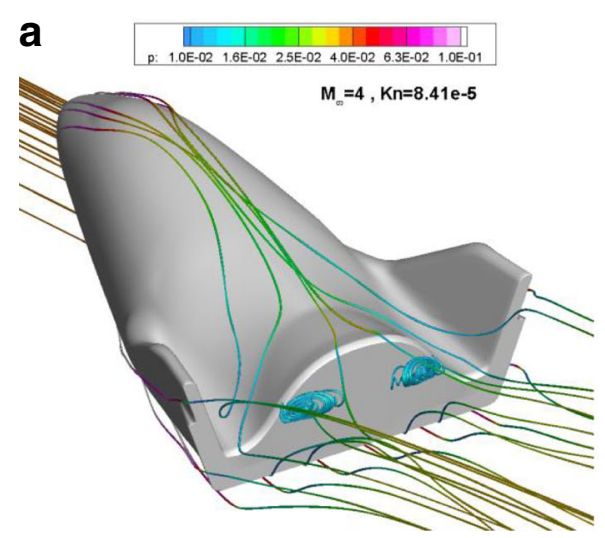

$\mathrm{M}=4, \mathrm{Kn}=8.41 \mathrm{e}-5$

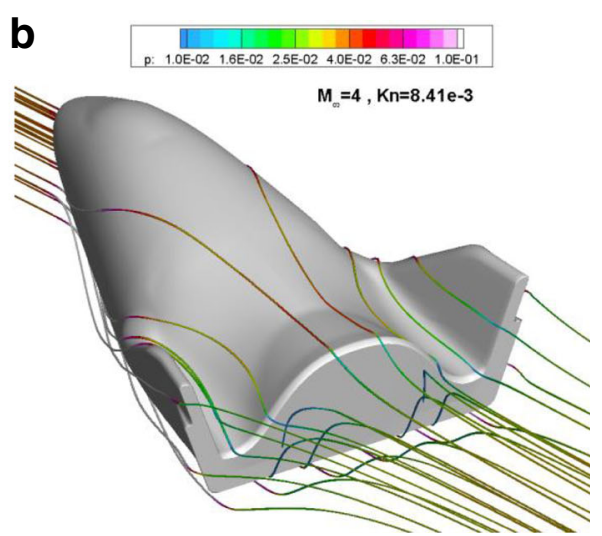

$\mathrm{M}=4, \mathrm{Kn}=8.41 \mathrm{e}-3$

Fig. 28 Spatial streamlines around the $X 38$-like vehicle (a) $M=4, K n=8.41 e-5$ (b) $M=4, K n=8.41 e-3$ 




four to five order of magnitude difference. Thus, such a multi-scale method as UGKS is needed in order to correctly simulate these flow fields.

Table 5 gives the aerodynamic coefficients comparisons for Mach number 8. The DSMC results are provided with RariHV which is an in-house DSMC software based on unstructured mesh in our group. The maximum relative error is only $2.27 \%$.

\section{Conclusions}

Our UGKS solver package is introduced including the main numerical techniques for improving the efficiency and accuracy, such as implicit method and local mesh refinement technique in the velocity space. It is devised for simulating flow fields around complex configurations for all flow regimes.

Several validations are conducted by comprehensive comparisons with industrystandard DSMC code and experimental results including the pressure, heat flux, shear stress and aerodynamic coefficients for supersonic and hypersonic flows at almost all regimes. The agreements are satisfactory in all cases.

Future work include more application to 3D complex configurations and complex flow, improvement on physical models to consider vibrational degree, implementation of models for gas mixtures, and increases in computational efficiency and accuracy.

Table 5 Comparisons of X38-like coefficients with Mach number 8

\begin{tabular}{lllll}
\hline Coefficients & $\mathrm{Kn}$ & UGKS & RariHV & Relative error(\%) \\
\hline Lift & $1.68 \mathrm{e}-1$ & $1.98 \mathrm{E}-01$ & $1.96 \mathrm{E}-01$ & 0.98 \\
& $1.68 \mathrm{e}-2$ & $1.94 \mathrm{E}-01$ & $1.92 \mathrm{E}-01$ & 1.15 \\
& $1.68 \mathrm{e}-3$ & $1.93 \mathrm{E}-01$ & $1.94 \mathrm{E}-01$ & -0.69 \\
Drag & $1.68 \mathrm{e}-1$ & $1.00 \mathrm{E}+00$ & $9.79 \mathrm{E}-01$ & 2.27 \\
& $1.68 \mathrm{e}-2$ & $5.56 \mathrm{E}-01$ & $5.46 \mathrm{E}-01$ & 1.85 \\
& $1.68 \mathrm{e}-3$ & $2.90 \mathrm{E}-01$ & $2.95 \mathrm{E}-01$ & -1.71 \\
\hline
\end{tabular}




\section{Acknowledgements}

The authors would like to thank professor Kun Xu for his help in the code development in the past 8 years and his advice on preparing the manuscript.

Funding

This work was supported by the National Natural Science Foundation of China (11402287 and 11372342).

\section{Availability of data and materials}

All data generated or analysed during this study are included in this published article.

\section{Authors' contributions}

DWJ programmed the whole code and conducted the UGKS simulations, and was a major contributor in writing the manuscript. MLM devised the code framework and guided the programming. JL conducted the DSMC simulations. XGD suggested some validation cases and analyzed the related results. All authors read and approved the final manuscript.

\section{Competing interests}

The authors declare that they have no competing interests.

\section{Publisher's Note}

Springer Nature remains neutral with regard to jurisdictional claims in published maps and institutional affiliations.

\section{Author details}

${ }^{1}$ Computational Aerodynamics Institute, China Aerodynamics Research and Development Center, Mianyang 621000,

China. ${ }^{2}$ National University of Defense Technology, Changsha 410000, China.

Received: 17 January 2019 Accepted: 22 January 2019

Published online: 13 March 2019

\section{References}

1. Ivanov MS, Gimelshein SF (1998) Computational hypersonic rarefied flows. Annu Rev Fluid Mech 30:469-505

2. Bird GA (1963) Approach to translational equilibrium in a rigid sphere gas. Phys Fluids 6(10):1518-1519

3. Bird GA (1990) Application of the direct simulation Monte Carlo method to the full shuttle geometry. AIAA Paper $90-1692$

4. Pham-Van-Diep G, Erwin D, Muntz EP (1989) Nonequilibrium molecular motion in a hypersonic shock wave. Science 245:624-626

5. Bird GA (2005) The DS2V/3V program suite for DSMC calculations. Rarefied Gas Dynamics. Amer Inst Physics, Melville, $541-546$

6. LeBeau GJ (1999) A parallel implementation of the direct simulation Monte Carlo method. Comput Methods Appl Mech Eng 174:319-337

7. Ivanov MS, Markelov GN, Gimelshein SF (1998) Statistical simulation of reactive rarefied flows: numerical approach and applications. AIAA Paper 98-2669

8. Dietrich S, Boyd ID (1996) Scalar and parallel optimized implementation of the direct simulation Monte Carlo method J Comput Phys 126(2):328-342

9. Scanlon TJ et al (2010) An open source, parallel DSMC code for rarefied gas flows in arbitrary geometries. Comput Fluids 39(10):2078-2089

10. Bhatnagar PL, Gross EP, Krook M (1954) A model for collision processes in gases I: small amplitude processes in charged and neutral onecomponent systems. Phys Rev 94(3):511-525

11. Shakhov E (1968) Generalization of the Krook kinetic equation. Fluid Dynamics 3(5):95-96

12. Rykov VA (1975) A model kinetic equation for a gas with rotational degrees of freedom. Fluid Dynamics 10(6):959-966

13. Titarev V, Dumbser M, Utyuzhnikov S (2014) Construction and comparison of parallel implicit kinetic solvers in three spatial dimensions. J Comput Phys 256:17-33

14. Wadsworth DC et al (2009) Assessment of Translational Anisotropy in Rarefied Flows Using Kinetic Approaches. Rarefied Gas Dynamics. Amer Inst Physics, Melville, 206-211

15. Mieussens $L$ (2000) Discrete-velocity models and numerical schemes for the Boltzmann-BGK equation in plane and axisymmetric geometries. J Comput Phys 162(2):429-466

16. Baranger C et al (2014) Locally refined discrete velocity grids for stationary rarefied flow simulations. J Comput Phys 257: 572-593

17. Li ZH, Zhang HX (2009) Gas-kinetic numerical studies of three-dimensional complex flows on spacecraft re-entry. J Comput Phys 228(4):1116-1138

18. Peng A-P et al (2016) Implicit gas-kinetic unified algorithm based on multi-block docking grid for multi-body reentry flows covering all flow regimes. J Comput Phys 327:919-942

19. Li Z et al (2015) A massively parallel algorithm for hypersonic covering various flow regimes to solve Boltzmann model equation. Acta Aeronautica et Astronautica Sinica 36(1):201-212

20. Xu K, Huang JC (2010) A unified gas-kinetic scheme for continuum and rarefied flows. J Comput Phys 229(20):7747-7764

21. Xu K (2015) Direct modeling for computational Fluid dynamics. World Scientific, Singapore

22. Huang JC, Xu K, Yu P (2012) A Unified Gas-Kinetic Scheme for Continuum and Rarefied Flows II: Multi-Dimensional Cases. Communications in Computational Physics 12(3): 662-690.

23. Jiang $D$ et al (2015) Study on the numerical error introduced by dissatisfying the conservation constraint in UGKS and its effects. Chinese J Theor Appl Mech 47(1):163-168

24. Titarev VA (2007) Conservative numerical methods for model kinetic equations. Comput Fluids 36(9):1446-1459 
25. Mao M et al (2015) Study on implicit implementation of the unified gas kinetic scheme. Chinese J Theor Appl Mech 47(5):822-829

26. Zhu Y, Zhong C, Xu K (2016) Implicit unified gas-kinetic scheme for steady state solutions in all flow regimes. J Comput Phys 315:16-38

27. Yu P (2013) A Unied Gas Kinetic Scheme For All Knudsen Number Flows. The Hong Kong University of Science and Technology, Hong Kong

28. Chen SZ et al (2012) A unified gas kinetic scheme with moving mesh and velocity space adaptation. J Comput Phys 231(20):6643-6664

29. Mao M (2006) Study of Practical Algorithm for numerical Simulation of Complicated hypersonic Flow. Dissertation, China Aerodynamics Research and Development Center.

30. Bird GA (1994) Molecular gas dynamics and the direct simulation of gas flows. Oxford Univ. Press,Inc, New York

31. Lofthouse AJ (2008) Nonequilibrium hypersonic aerothermodynamics using the Direct Simulation Monte Carlo and Navier-Stokes models. Dissertation, University of Michigan

32. Li J et al (2018) Novel hybrid hard sphere model for direct simulation Monte Carlo computations. J Thermophys Heat Transf 32(1):156-160

33. Wendt JF (1971) Drag Coefficients of Spheres in Hypersonic Non-Continuum Flow. von Karman Institute for Fluid Dynamics, Belgium

34. Boyd ID, Chen G, Candler GV (1995) Predicting failure of the continuum FLUID equations in transitional hypersonic flows. Phys Fluids 7(1):210-219

Submit your manuscript to a SpringerOpen ${ }^{0}$ journal and benefit from:

- Convenient online submission

- Rigorous peer review

- Open access: articles freely available online

High visibility within the field

- Retaining the copyright to your article

Submit your next manuscript at $>$ springeropen.com 